\title{
EchoGéo
}

$21 \mid 2012$

Pays émergents

\section{Brigade Road et Church Street, Bengaluru (Inde) : des espaces publics où " l'Inde qui brille » se met en scène}

\section{Aurélie Varrel}

\section{CpenEdition}

\section{Journals}

Édition électronique

URL : https://journals.openedition.org/echogeo/13053

DOI : 10.4000/echogeo.13053

ISSN : 1963-1197

Éditeur

Pôle de recherche pour l'organisation et la diffusion de l'information géographique (CNRS UMR 8586)

Référence électronique

Aurélie Varrel, « Brigade Road et Church Street, Bengaluru (Inde) : des espaces publics où «I'Inde qui brille » se met en scène », EchoGéo [En ligne], 21 | 2012, mis en ligne le 10 octobre 2012, consulté le 10 août 2021. URL : http://journals.openedition.org/echogeo/13053 ; DOI : https://doi.org/10.4000/ echogeo.13053

Ce document a été généré automatiquement le 10 août 2021.

EchoGéo est mis à disposition selon les termes de la licence Creative Commons Attribution - Pas d'Utilisation Commerciale - Pas de Modification 4.0 International (CC BY-NC-ND) 


\section{Brigade Road et Church Street, Bengaluru (Inde) : des espaces publics où « l'Inde qui brille » se met en scène}

Aurélie Varrel

Illustration 1 - Brigade Road

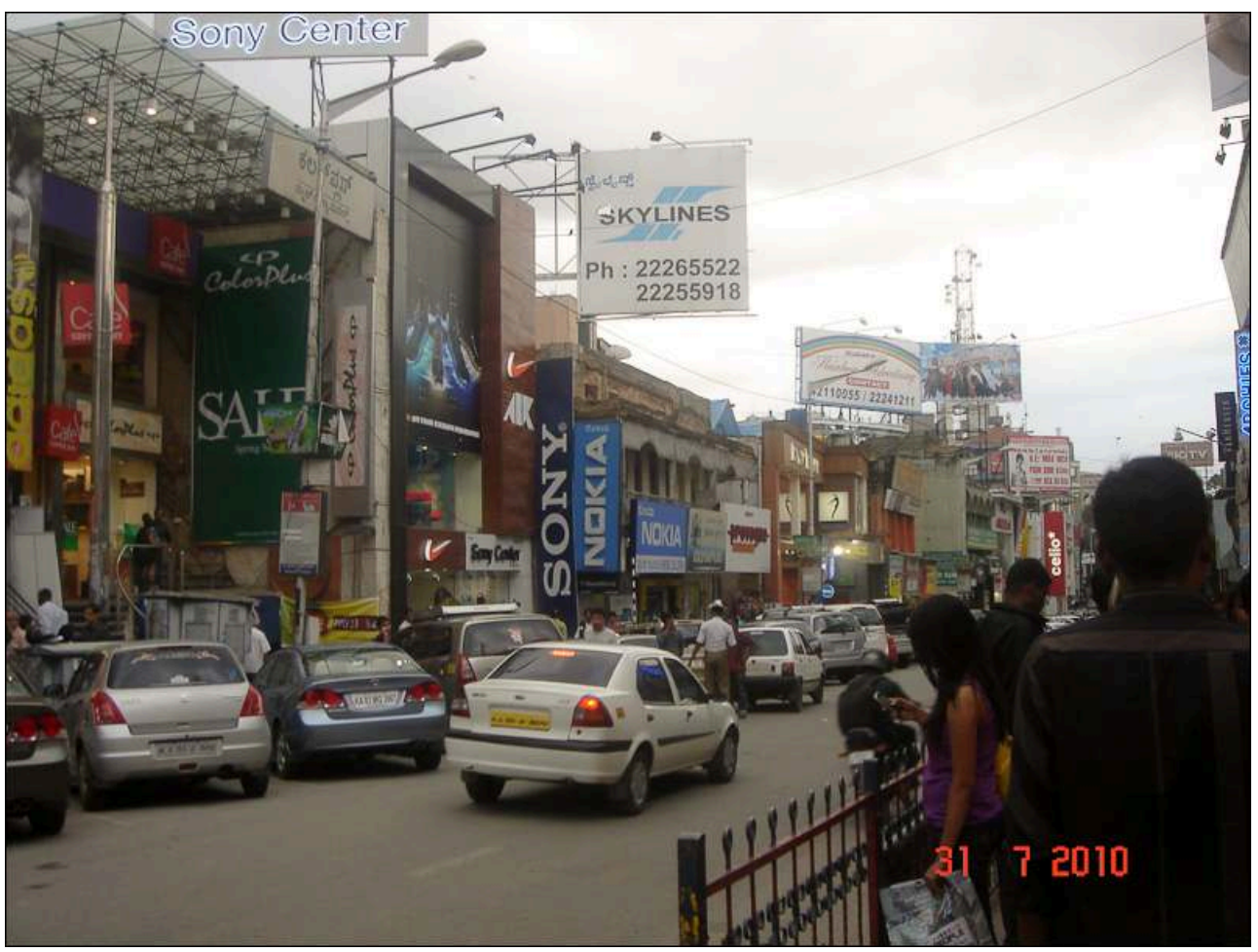

Auteur : Aurélie Varrel, 2010 


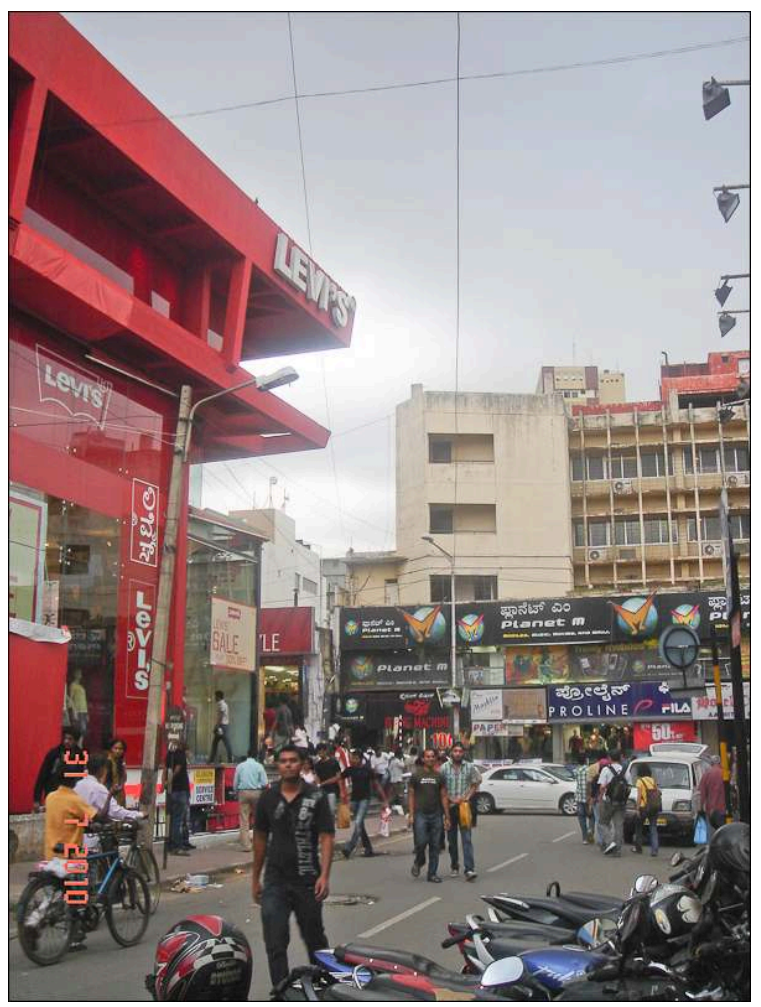

Auteur : Aurélie Varrel, 2010.

1 Brigade Road (illustration 1) et Church Street (illustration 2, intersection avec Brigade Road au second plan) sont deux rues situées dans le quartier commerçant de l'ancien noyau britannique de Bangalore, le cantonment.

2 Leurs noms et leurs paysages sont connus en Inde : elles constituent un espace vitrine où sont mis en scène les nouveaux modes de consommation et de vie ainsi qu'une certaine idée de la ville mondialisée qui se sont développés depuis 1991, date de lancement des mesures de libéralisation économique (Zérah, 2010). Les façades disparaissent sous l'habillage des devantures : on observe tout juste quelques éléments décoratifs typiquement coloniaux, au-dessus de la boutique Nokia, sur l'illustration 1. Enseignes et panneaux publicitaires renvoient autant à des marques multinationales (Nokia, Sony, Levis) qu'à des enseignes indiennes vendant des biens de consommation coûteux, comme la chaîne de magasins d'électronique, CD et DVD «Planet M » (en arrière-plan sur l'illustration 2). La voirie a été modifiée pour suivre un modèle européen, avec places de parking en épi et horodateurs importés. Les usages de l'espace public sont strictement régulés : des grilles, visibles sur l'illustration 1 , ont été posées pour contraindre les piétons à rester sur les trottoirs; seules les voitures et les motos ont désormais le droit de descendre Brigade Road, sans s'arrêter sauf place de parking libre ; un policier, reconnaissable à son chapeau blanc sur l'illustration 1, fait respecter ces dispositions exceptionnelles en Inde. Ces mesures excluent en particulier les innombrables rickshaws, mode de transport privilégié dans les villes indiennes, qui déposent ou prennent des passagers le long des rues. La construction de cet espace vitrine est donc autant le fruit des acteurs privés que d'une politique d'urbanisme public beaucoup plus volontariste que dans le reste de la ville. 
Ces espaces vitrines cristallisent aussi les tensions qui accompagnent le "grand écart " social, économique et culturel croissant (Landy, 2007) depuis 1991, entre une «Inde qui brille» et le reste du pays qui se développe plus lentement. Ainsi l'hebdomadaire Outlook a fait sa couverture en décembre 2007 sur une photo de Brigade Road en titrant : «Why Bangalore hates IT culture » [IT : Information Technology, c'est-à-dire le secteur informatique dont Bangalore est le premier pôle en Inde (Leducq, 2009)]. Si les évolutions que connaît Bangalore ne sauraient être réduites à son image savamment promue de "Silicon Plateau » de l'Inde, ces lieux servent à mettre en scène des styles vestimentaires et des manières d'être particuliers. Ces rues, leurs magasins ainsi que leurs pubs et restaurants sont des lieux de sociabilité à tous moments de la semaine pour la jeunesse aisée que l'on voit sur ces photos, étudiants et jeunes adultes, qui, les fréquentent habillée à l'occidentale, en exhibant des objets tels que téléphones portables dernier cri, motos coûteuses, etc. En fin de semaine, la fréquentation se diversifie et s'intensifie, et ce paysage lui-même devient facteur d'attraction pour une foule plus familiale et plus diverse venant se promener dans ce qui demeure un espace public ouvert, à la différence par exemple des malls aux entrées contrôlées.

\section{BIBLIOGRAPHIE}

Landy F., 2007. L'Inde ou le grand écart. Paris, La Documentation Photographique, ${ }^{\circ} 8060$.

LeducqD., 2009.La diffusion spatiale de l'informatique en Inde; EchoGéo [En ligne], Numéro 10 | 2009, mis en ligne le 03 septembre 2009. URL : http://echogeo.revues.org/11355

Zérah M.-H., 2009. Une « Vision Mumbai » pour transformer la ville ou la difficulté à (re)penser la gouvernance métropolitaine. EchoGéo [En ligne], Numéro $10 \mid$ 2009, mis en ligne le 10 septembre 2009. URL : http://echogeo.revues.org/11389

\section{AUTEUR}

\section{AURÉLIE VARREL}

Aurélie Varrel, aurelie.v@gmail.com, est chargée de recherche CNRS en géographie au Centre d'Etude de l'Inde et de l'Asie du Sud (UMR 8564 CNRS-EHESS) (http://ceias.ehess.fr/ document.php?id=1436). Elle a publié récemment :

- Varrel A., 2011. Migration in the light of the new Indian diaspora policy: emerging transnationalism. In Rajan Irudaya, Percot Marie (dir.), Dynamics of Indian Emigration. Historical and current Perspectives, Delhi, Routledge, 2011.

- Varrel A., 2011. Gender and intergenerational issues in the circulation of high skilled migrants. The case of the Indian IT professionals . In Kofman E., Kohli M., Kraler A., Schmoll C. (dir.), Gender, Generations and the Family in International Migration, Amsterdam, Free University Press, 2011, p. 243-257.

- Varrel A., 2010. Notices : « Bangalore », « NRI », « Karnataka », « NASSCOM », « immobilier », 
« centre d'appel ». In Landy Frédéric (dir.). Dictionnaire de l'Inde contemporaine, Paris, Armand Colin, 2010. 